\title{
The Sub-critical Flow past a Generic Side Mirror and its Impact on Sound Generation and Propagation
}

\author{
Jonas Ask * \\ Volvo Car Corporation, Fluid Dynamic Center SE-405 31 Göteborg, Sweden \\ and Lars Davidson ${ }^{\dagger}$ \\ Chalmers University of Technology, SE-412 96 Göteborg, Sweden
}

\begin{abstract}
The investigated case is the flow past a generic side mirror mounted on a flat plate at the Reynolds number of $R e_{D}=5.2 \cdot 10^{5}$ based on the mirror diameter. The flow field over the mirror is identified as sub-critical which makes LES an attractive candidate to the DES model used by some of the previous researchers. The present paper is a first attempt to investigate the flow and acoustics of a generic side mirror at the Reynolds number of $R e_{D}=5.2 \cdot 10^{5}$ by comparing DES and LES results using identical grid, second order schemes and the incompressible assumption. Two different cases are investigated for this purpose. The first case uses the hybrid DES-modelling technique combined with a "Trip-Less" approach proposed by Shur et al. and the second case is a partially resolved LES-simulation using the dynamic Smagorinsky model. The sound radiation for the present simulations is predicted from the Ffowkes-Williams and Hawkings analogy where the wall pressure fluctuations and the pressure temporal derivative are the two source terms treated. The result shows good agreement for both cases in wall source levels even with the rather coarse mesh used in the mirror wake. The trends are however in slightly better agreement for the LES case than the DES case compared to the corresponding measured levels. The radiated sound for the DES case agrees significantly better with the measured results compared to the LES results. The overprediction in radiated sound observed in the LES case is argued to primarily be caused by unresolved fluctuations included in the surface integration. The DES model on the other hand is far more dissipative and reduces pollution of numerical noise in the signals. For an automotive application the wall pressure level results is by far the most important due to the interest in the driver and passengers subjective experience. Thus, the conclusion is that the LES approach is a better candidate compared to DES even though the mesh resolution over the plate by no means fulfills the traditional restrictions in LES.
\end{abstract}

\section{Introduction}

Flow induced noise experienced by the driver or passengers of a ground vehicle can be a consequence of different flow or fluid-structure phenomena occurring in low Mach number flows. Exposed components such as side mirrors, A-posts, rails, tires and underfloor details generate flow structures, which are the primary sources to noise generation around the vehicle above approximately $120 \mathrm{~km} / \mathrm{h}$. The present work studies the flow past a generic side mirror mounted on a flat plate with respect to sound generation and propagation. This geometry has been the subject of several studies [1-5]. Höld and Siegert [1,2] conducted both experimental and numerical investigations to predict sound generation and propagation at the Reynolds number of $R e_{D}=7.066 \cdot 10^{5}$ based on the mirror diameter. They conducted URANS simulations for three different mesh densities and used an acoustic analogy to compute the radiated sound based on the wall pressure fluctuations and its temporal derivative $[6,7]$. The simulation time in their work covered 0.1

\footnotetext{
*PhD student

${ }^{\dagger}$ Professor, Division of Fluid Dynamics, Department of Applied Mechanics

Copyright (C) 2006 by J. Ask, L. Davidson . Published by the American Institute of Aeronautics and Astronautics, Inc. with permission.
} 
seconds. A similar study at the Reynolds number of $R e_{D}=5.2 \cdot 10^{5}$ was reported in Rung et al. [3] which focused on differences in sound generation and propagation in comparisons of URANS and the Detached Eddy Simulation (DES) modeling technique, Spalart et al. [8]. The maximum simulation time in their study covered 0.26 seconds, and the grid density was twice the highest resolved case in Siegert et al.[1,2]. Results of Rung's DES simulation generally showed an overprediction of the low frequency fluctuating pressure levels for most surface mounted sensors and microphones. Their findings showed however a major improvement in acoustic results using the DES model as compared with the URANS results. The code vendor EXA later used a generic side mirror to validate their CAA method development in an aeroacoustic consortium directed toward car manufacturers worldwide. They used the same Reynolds number as Rung et al. but avoided the difficulties associated with detailed separation control by introducing a "trip wire" in the simulations that was not present in the experiments. Their simulation covered 0.67 seconds and showed the same trends as reported in Rung et al. but had improved high frequency contents. The frequency bin width for their simulation was $19.5[\mathrm{~Hz}]$. Another code vendor, FLUENT, followed the experiment reported in $[1,2]$ at the higher Reynolds number. Their prediction of the radiated sound past the mirror was promising, but confusing figures raise the question of where the monitoring points were actually located.

The present paper is a first attempt to investigate the flow and acoustics of a generic side mirror at the Reynolds number of $R e_{D}=5.2 \cdot 10^{5}$ by comparing DES and LES results using identical grid.

Several authors have previously reported promising results for similar geometries and flow fields with the DES model [9-14]. In contrast to previous simulations conducted over a generic side mirror, a "trip-less" approach is chosen for the DES case to capture an eventual laminar separation over the front side of the mirror. This methodology was pioneered by Shur et al. [15] and has been further investigated in $[9,10,14,16]$ for cylinders and spheres. Shur and Spalart [15] used this methodology for URANS simulations in two dimensions past a circular cylinder, and three-dimensional investigations were conducted by Travin, Strelets and Constantinescu et al. [9, 10, 14].

In the LES case we use the dynamic Smagorinsky model pioneered by Germano et al. [17] and Lilly [18].

Concerning sound radiation, the most straightforward way is to conduct a compressible direct simulation (DS). With this approach it is possible to directly evaluate sound emissions at any position in the computational domain. This technique puts however enormous constraints on numerical schemes and boundary conditions in order to capture the wide range scales present in any turbulent flow field. If interest is instead in finding the emissions at points outside the computational domain or from an incompressible flow field, a different strategy must be used. For flows where walls are present, a feasible method is to use either Ffowkes-Williams and Hawkings or Lighthill-Curle's analogy $[6,19]$. These analogies convert the governing momentum and continuity equations to an inhomogeneous wave equation and are identical for steady and impermeable integration surfaces. Two major assumptions are made in these analogies:

- The fluctuating density, which is the dependent variable, exist on both sides of the equation and thus decouples the sound wave operator from the sources. This is a reasonable assumption for low Mach number flows, where sound emissions are primarily caused by the hydrodynamics.

- Only isotropic wave propagation is taken into account, which is true only for flows with zero mean motion.

Studies of the incompressible assumption combined with a modified version of Lighthill-Curle's analogy were presented in Ask and Davidson [20,21]. The incompressible assumption is controversial and strictly speaking not valid for any flow field, but the results were promising in terms of both directivity and sound pressure levels. The modified version of Lighthill-Curle's analogy, Eq. 1, uses the temporal derivatives inside the integral instead of keeping the spatial derivatives outside the integral, as Curle's [19] original formulation states.

$$
\begin{aligned}
& p(\mathbf{x}, t)-p_{\infty}= \\
& \frac{1}{4 \pi} \int_{V}\left[\frac{l_{i} l_{j}}{a_{\infty}^{2} r} \ddot{T}_{i j}+\frac{3 l_{i} l_{j}-\delta_{i j}}{a_{\infty} r^{2}} \dot{T}_{i j}+\frac{3 l_{i} l_{j}-\delta_{i j}}{r^{3}} T_{i j}\right] d V(\mathbf{y}) \\
& +\frac{1}{4 \pi} \int_{S} l_{i} n_{j}\left[\frac{\dot{p} \delta_{i j}-\dot{\tau}_{i j}}{a_{\infty} r}+\frac{p \delta_{i j}-\tau_{i j}}{r^{2}}\right] d S(\mathbf{y})
\end{aligned}
$$

In Eq. 1 the acoustic pressure fluctuations at an observer located at $\mathbf{x}$ are evaluated from a forward time projection of two integrals evaluated at the source location, $\mathbf{y}$. The first integral contains the volume 
contribution where $T_{i j}=\rho u_{i} u_{j}-\tau_{i j}+\left(p-a_{\infty}^{2} \rho\right) \delta_{i j}$ is commonly referred to as the Lighthill tensor. The $\operatorname{dot}(\mathrm{s})$ above $T_{i j}, \tau_{i j}$ and $p$ denote time derivative(s), and $l_{j}$ is the unit vector pointing from the source to the observer; the terms in this integral are often referred to as quadrapole terms. The second integral contains the surface integral and consists mainly of the fluctuating pressure and the fluctuating pressure temporal derivative. These terms are commonly referred to as dipole terms and are often regarded as the dominating terms for low Mach number wall bounded flows. The derivation of the expression above can be found in [22] and the corresponding version of Ffowkes-Williams and Hawkings equation can be found in Brentner and Farassat [7] for the conditions mentioned above. A reduced version is also implemented in the current version of FLUENT where the pressure dipole terms are the two terms kept.

The methodology for evaluating the radiated sound involves two steps. First, the flow field is computed on the basis of the assumption of incompressibility. From the flow field obtained, the fluctuating wall pressures and their temporal derivative are extracted and used in a second step to compute the radiated sound $[6,7,20-22]$. In this work the volume sources are neglected owing to the storage cost. This means that the fluctuating wall pressure and the time derivative of the fluctuating wall pressure are the only terms treated.

The generic side mirror is geometrically similar to both the sphere and the cylinder, perhaps best known for their drag crisis around a critical Reynolds number. This great difference in drag force is the consequence of laminar versus turbulent separation over the object. The Reynolds number for the present simulation is $\operatorname{Re}_{D}=5.2 \cdot 10^{5}$, which is close to the critical Reynolds number for both the sphere and the cylinder. According to Schlichting [23], the drag force coefficient over a circular cylinder drops 0.7 over the interval $3.0 \cdot 10^{5}<R e_{D}<5.0 \cdot 10^{5}$. Similar results can also be found for the sphere, where the force coefficient drops 0.31 over the interval $2.5 \cdot 10^{5}<R e_{D}<4 \cdot 10^{5}$. Constantinescu and Squires [13] mention a critical Reynolds of $R e_{c r} \approx 3.7 \cdot 10^{5}$ for the sphere, which agrees well with Schlichting [23]. If an analogy is made between the flow state over the side mirror and the corresponding sphere and cylinder, the boundary layer over the generic side mirror in the present state is close to its critical value. An issue that emphasizes this problem is two different experimental results for the same flow conditions. The first is the work presented by Rung et al. [3], who state:

"Oil-flow visualization revealed that flow is clearly super-critical, with transition occurring approximately $0.15 \mathrm{D}$ upstream of rear-face edge."

Secondly, personal communication and results obtained from Daimler-Chrysler [24] showed that subsequent measurements indicate, in contradiction with the annotation above, that the flow state over the mirror is actually sub-critical.

On the basis of this information it was further assumed that the flow state over the mirror is actually sub-critical, with an upstream laminar separation. This makes LES a promising candidate to DES due to the absence of near wall streaks present in the turbulent shear layer. Or in other words, the resolution in the spanwise direction over the mirror front side can be coarser compared to what is traditionally acceptable for turbulent shear layers.

As regards spatial discretization of the convective fluxes for DES, a general recommendation was made by Strelets [10] and further applied in $[13,14,25,26]$. He suggested central difference schemes in the wake combined with high-order, upwind-biased schemes in the RANS and Euler regions if the pure central differenced scheme proved unstable. The central schemes are known to have stability issues when operating in RANS mode, especially for cells with large aspect ratios. On the other hand, upwind-biased schemes are commonly considered too dissipative for LES. Different spatial discretization practices have been used in the literature in connection with DES. In most extreme cases, a fifth-order, upwind-biased scheme was applied in the Euler and RANS region combined with a fourth-order central scheme in the wake [25,26]. In $[13,14]$, a fifth-order upwind scheme was used close to the walls and over the Euler region and was combined with a second-order central difference scheme in the wake region. However, several authors have reported acceptable results also for second and third-order, upwind-biased schemes [3,12] involving separated flows.

The present paper is organized as follows. Section II describes the numerical approach used in the present simulations. This is followed by the result sections which consist of flow field results (Section III) and acoustic results (Section IV). Conclusions are presented in Section V and acknowledgments are stated in Section VI. One appendix is also included giving the locations of pressure sensors and microphones (Appendix A). 


\section{Numerical Approach}

The object used in the present simulation is a generic side mirror mounted on a flat plate. It consists of a half cylinder with the diameter $D=0.2[\mathrm{~m}]$ and height $H=D$ blunted by a quarter sphere on top, which gives a total height of $H=1.5 D$. The geometry is shown in Fig. 1 and its position on the plate is represented in Fig. 2. The upstream part of the mirror will in the following text be referred to as the front side and downstream flat side will be referred to as the rear side. The Reynolds number based on the mirror diameter is $R e_{D}=5.2 \cdot 10^{5}$ with a freestream velocity of $39[\mathrm{~m} / \mathrm{s}]$. This freestream velocity results in a Mach number of 0.11 . There are no indications that compressible effects nor sound wave reflections have a prominent role in the present flow field, which implies that an incompressible approach should be justified.
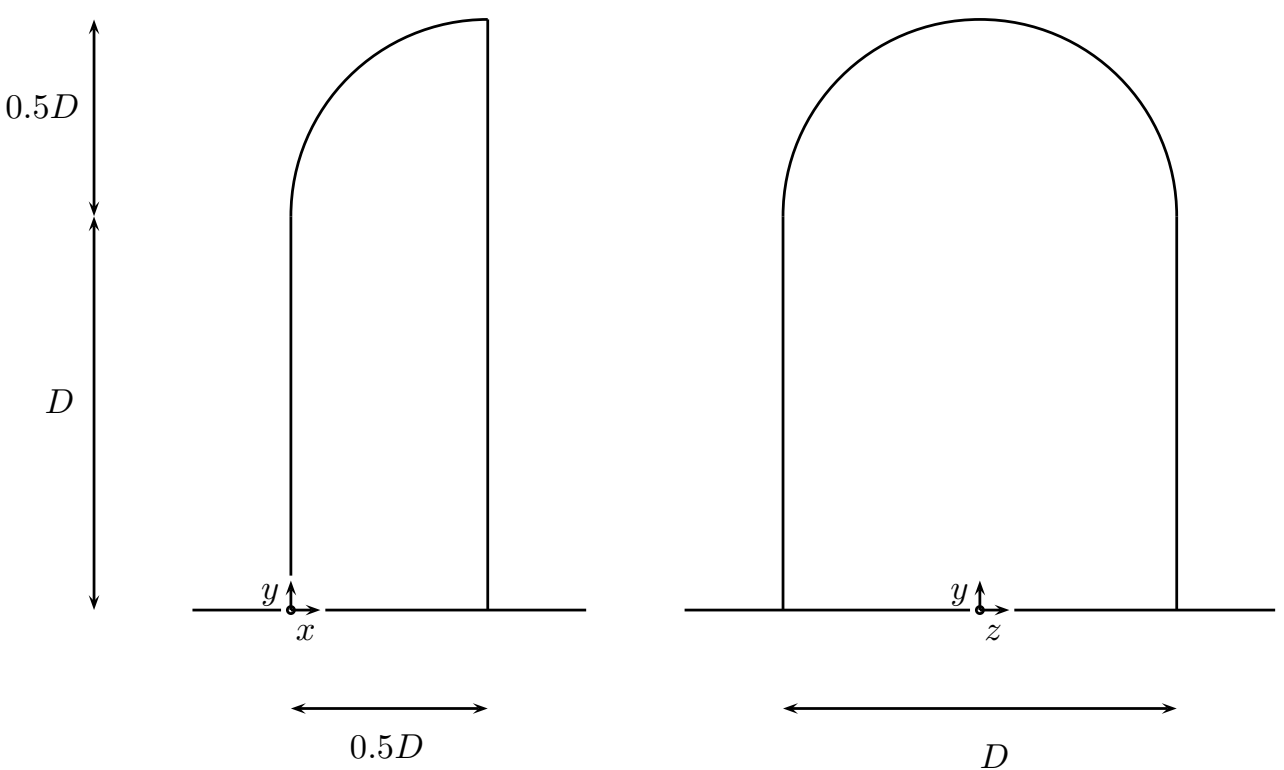

Figure 1. Mirror geometry; side view and front view, respectively

The code used in this paper is the unstructured FLUENT 6.2 commercial solver. It is based on a control volume formulation to convert the governing equations to a solvable set of algebraic equations. The code in the present state uses a collocated scheme with cell-centered storage of both scalars and vectors. In the present paper, the incompressible segregated solver is used with an implicit SIMPLEC pressure-velocity coupling algorithm. The discretization schemes for the convective fluxes are a second-order, upwind-biased scheme for the DES case and a Bounded Central Difference (BCD) scheme for the LES case. The BCD scheme is essentially a second order central scheme with a wiggle detector for wave lengths of $2 \Delta x$ or less. For these occasions it blends with a second-order upwind scheme or in worst case a first-order upwind scheme. The discretization in time follows an implicit second-order scheme to obtain a converged solution, and convergence is defined as when the residuals have dropped three orders of magnitude each time step for the solved variables.

The dynamic Smagorinsky constant $C_{s}$ is clipped at 0 and 0.23 and the test-filter comprises the computational cell and the neighboring cells that share the same faces. For hexahedral cells used in the present work the ratio between test-filter and grid-filter is approximately 2.1 or $9^{1 / 3}$.

The computational domain is built up by a block-structured hexahedral mesh in ICEM 5.1 and consists of $N_{x y z} \approx 5.83 \cdot 10^{6}$ cells. The domain is bounded by an inlet $15 D$ upstream of the mirror and an outlet $30 D$ downstream of the mirror. In the spanwise extension the farfield boundaries are positioned $15 D$ on opposite 
sides of the mirror. In the plate normal direction a farfield boundary is located $15 D$ above the plate.

The inlet boundary condition for the DES case follows the "trip-less" approach, with an initial steady state inlet turbulence intensity of $0.1 \%$ and a turbulent inlet length scale of $0.5 \mathrm{D}$ followed by a zero inlet turbulent viscosity. For the LES case no perturbations are applied at the inlet. Other boundary conditions used in the present work are pressure boundary condition at the outlet and symmetry boundary conditions at the farfield boundaries. In this text $n^{+}$will refer to the wall normal direction, $s^{+}$to the streamwise direction and $l^{+}$to the lateral direction. At solid boundaries the log-law is applied for cells located in the range of $30<n^{+}<300$ and the viscous sublayer is assumed to be resolved for cell centroids located at $n^{+}<5$. In the intermediate region a blending of the wall shear stress is carried out. An attempt to resolve the boundary layer over the plate would increase the amount of cells necessary beyond the limit of available computer resources due to the resolution restriction in all spatial directions. The log-law is therefore assumed to be valid over a major part of the plate while resolving the viscous sublayer over the front side of the mirror.

After a total simulation time of $97.5 \mathrm{D} / \mathrm{U}$, extraction of statistics and wall pressures was initiated for the DES case and the simulation was run for a further $101.4 D / U$. The DES case served then as an initial solution to the LES case and was run for a further $71 D / U$ before initiating extraction of the statistics. Then the simulation was run for a further $117 D / U$. The time step for both cases was $\Delta t=2 \cdot 10^{-5}[\mathrm{~s}](=T U \cdot D / U)$, where $\mathrm{TU}$ is Time Units $(T U=D / U \approx 0.005[\mathrm{~s}])$.

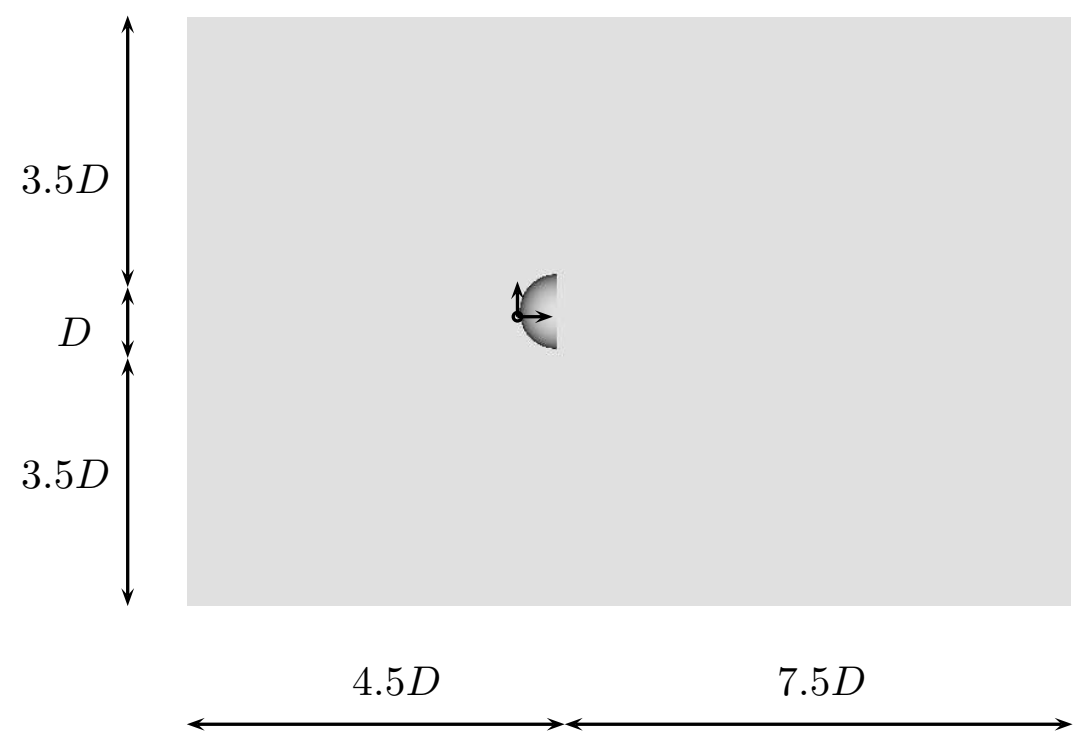

Figure 2. Mirror and plate, $x z$-plane

The front side of the mirror is resolved by 7900 cells with 105 nodes over the mirror height and 55 cells over the mirror width. The first off-wall node close to the mirror trailing edge is located at $\Delta y=2 \cdot 10^{-2}[\mathrm{~mm}]$ in the wall normal direction. As an estimate of the boundary layer thickness, a laminar Blasius solution for the cylinder flow was used [23]. At a Reynolds number of $R e_{D}=5.2 \cdot 10^{5}$, the estimated boundary layer thickness, $\delta_{R=0.1} \approx 0.59[\mathrm{~mm}]$, is resolved by 10 cells in the wall normal direction. To prevent an eventual grid-induced separation in the DES case, the following relation must be fulfilled, Menter et al. [27]:

$$
C_{D E S} \Delta>\mathcal{O}(1) \delta
$$

In Eq. $2, \Delta$ is the maximum extension of the cell, $C_{D E S}=0.65$ and $\delta$ is the boundary layer thickness. For the cells located over the front side close to the trailing edge, this requirement is fulfilled by at least one order of magnitude.

\section{Flow field results}

This section gives flow field results for the two simulations. The results are further divided into mean and instantaneous results, followed by the time-dependent results. 


\section{A. Mean and instantaneous results}

The $n^{+}$values for the DES and LES case ranges from $0.19<n^{+}<5.4$ and $0.09<n^{+}<7.0$ respectively over the mirror front side. Over the plate, the $n^{+}$values for both cases typically range from 0.8 to 410 , where the maximum values occurs close to the leading edge of the plate and the lower limit values typically occurs locally at spots in the mirror wake. Snap-shots of the $n^{+}$levels over the plate are also shown in Fig. 3 . The $s^{+}$is typically $34-37$ on the front side of the mirror close to the trailing edge and the corresponding $l^{+}$value varies between 130 and 150 for the same locations.

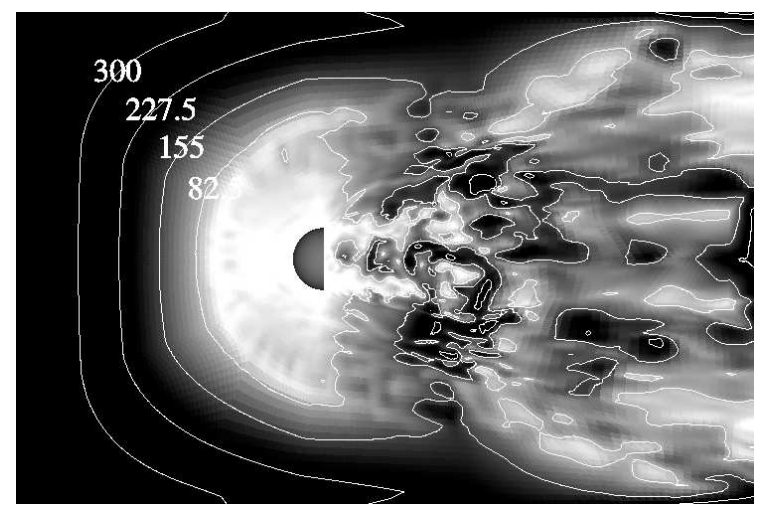

(a) Contour levels of $n^{+}$over the plate, LES

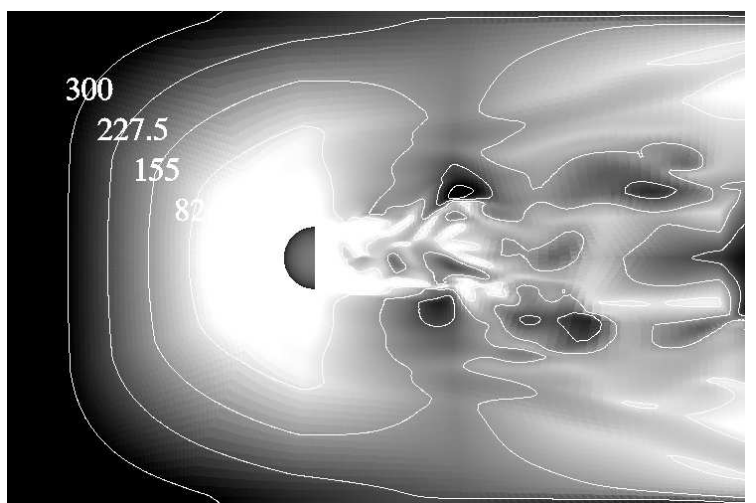

(b) Contour levels of $n^{+}$over the plate, DES

Figure 3. Contours of instantaneous $n^{+}$over the plate

The CFL number ranges from $0.0014<C F L_{\text {conv }}<2.85$ for the DES case and $0.0016<C F L_{\text {conv }}<11.06$ for the LES case where the maximum value typically occurs close to the mirror trailing edge and is limited to just a few cells.

From the oil film visualization picture Fig. 4(a), an upstream separation line can clearly be identified located approximately $0.15 \mathrm{D}$ measured from the rear side edge. An instantaneous LES result of the wall shear stress is also presented in Fig. 4(b) and indicates a separation which is in fair agreement with the measurement. The DES case lacks this feature completely, Fig. 5(a). The reason can be understood from Fig. 5(b) where an excessive production of turbulent viscosity prevents the separation to occur.

Figures 6 shows the location of the RANS/LES switch over the $z=0$ plane in comparison to the wall normal grid. A close-up view over the trailing edge is seen in Fig. 6(b) together with superimposed velocity vectors. As discussed above, the boundary layer is completely captured within the RANS domain even though the $s^{+}$and $l^{+}$are significantly lower than what is customary for a low Reynolds RANS grid.

Figure 7 shows the location of the mean pressure sensors. The mean pressure for these locations are presented in Fig. 8 and shows measured results [24] as well as the two conducted simulations. Sensors $S 1-S 9$ are located downstream the separation line identified from the measurements and both simulations underpredicts the mean pressure slightly at these locations. This underprediction is even more pronounced at the upstream positioned sensors $S 10$ and $S 25$ and is caused by a misrepresentation of the separation zone. The mean pressure over the mirror front side show otherwise good agreement with the measured results and on the rear side a perfect agreement between the LES case and the measurement is obtained. A complete description of the monitor positions is given in Table A in Appendix A.

\section{B. Time dependent results}

Mirror force coefficients are presented in Fig 9 and shows a higher mean level for the LES case compared to the DES case. The lower drag force obtained from the DES case is due to a higher pressure recovery over the mirror rear side as was identified from the mean pressure sensors in Fig. 8. Another finding shared by others using the DES model is the lack of high frequency content in the results. The flow field over the front side of the mirror is steady as compared to the rear side of the mirror. The low frequency content in the drag force is thus primarily due to a lack of smaller resolved scales in the wake, meaning that only large 


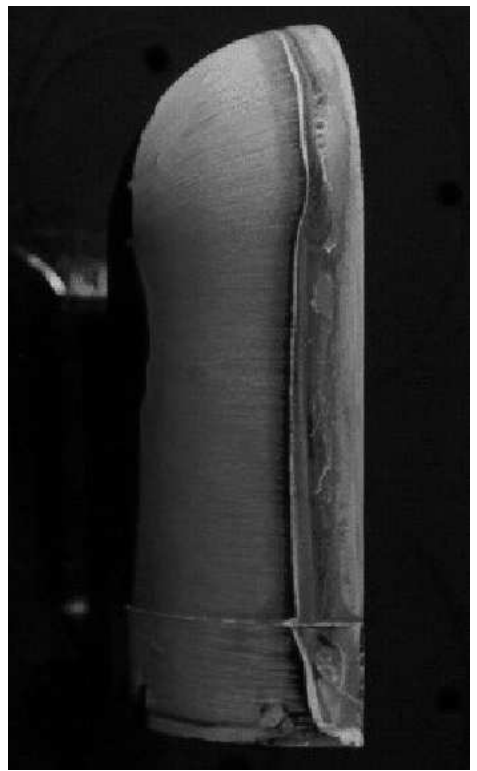

(a) Oil film visualization, (Courtesy of Daimler Chrysler Research and Technology)

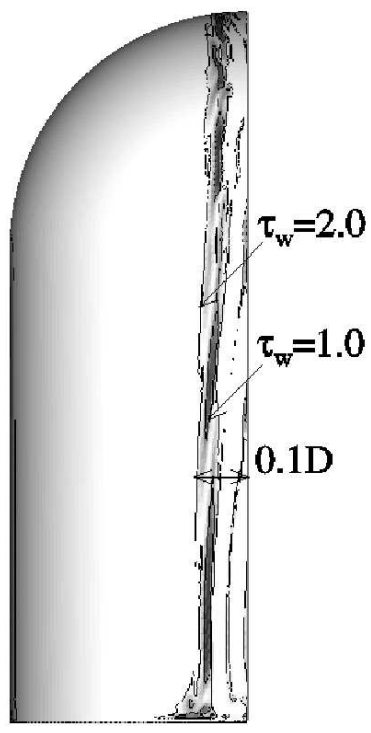

(b) Snapshot of wall shear stress, LES, $0<\tau_{\text {wall }}<2.0$, (decreasing in streamwise direction)

Figure 4. Oil film visualization and wall shear stress

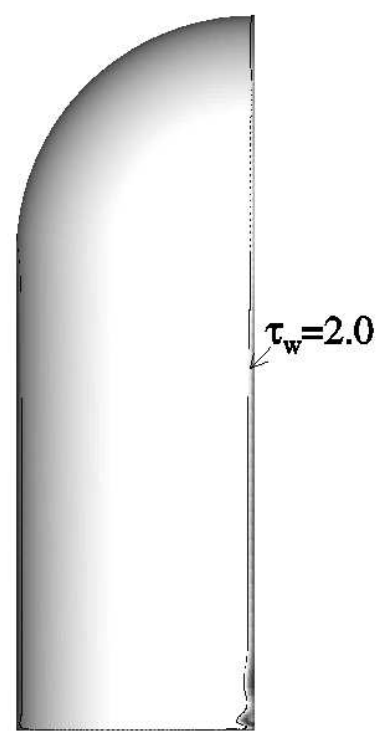

(a) Snapshot of wall shear stress, DES, $0<\tau_{\text {wall }}<2.0$, (decreasing in streamwise direction)

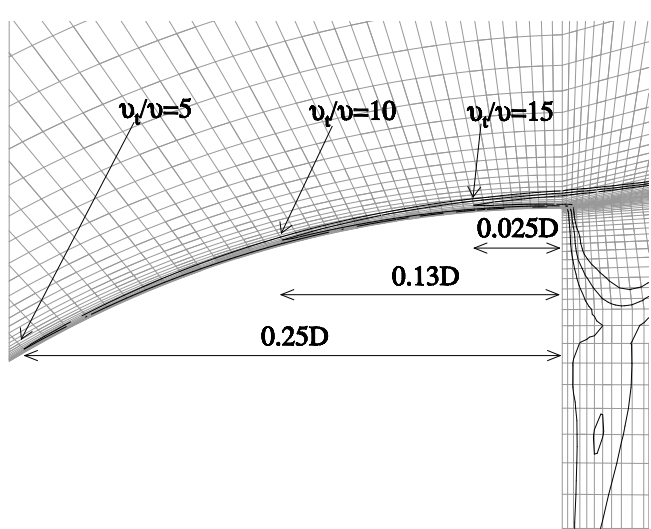

(b) Turbulent viscosity ratio over plane $z=0$, DES

Figure 5. Wall shear stress and turbulent viscosity ratio over plane $z=0$, DES 


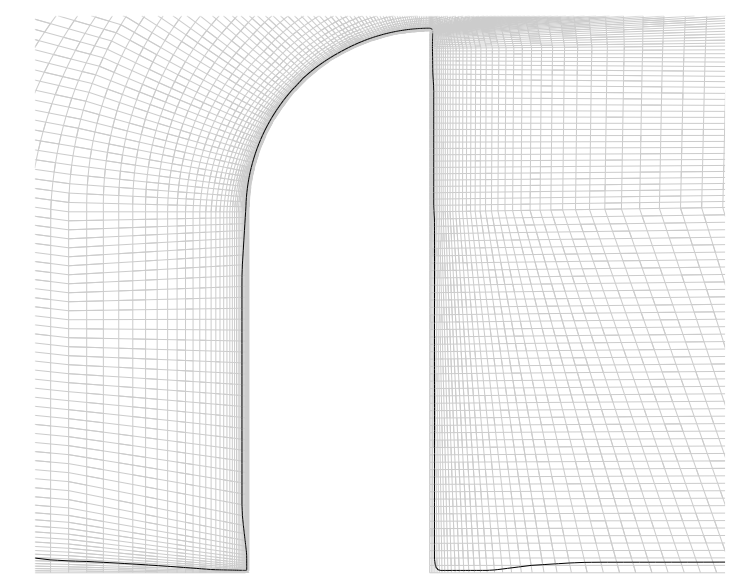

(a) Grid and location of the RANS/LES switch, DES

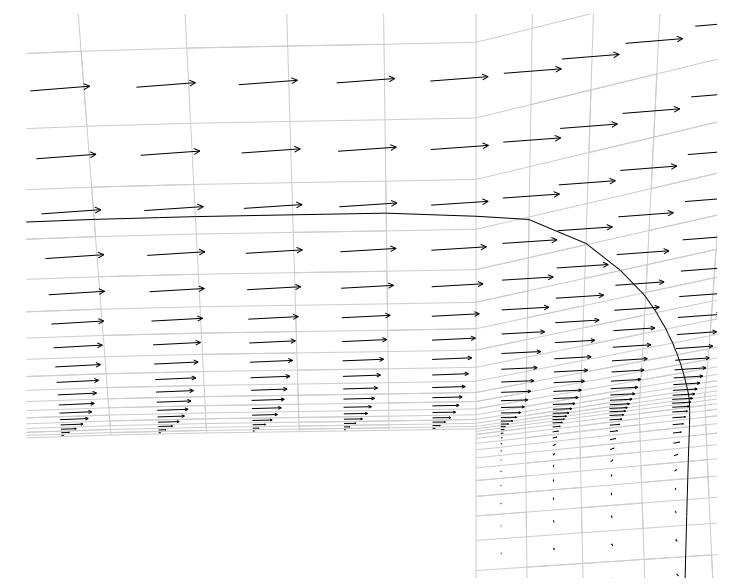

(b) Velocity vectors and location of the RANS/LES switch close to the trailing edge, DES

Figure 6. Grid and location of the RANS/LES switch, DES

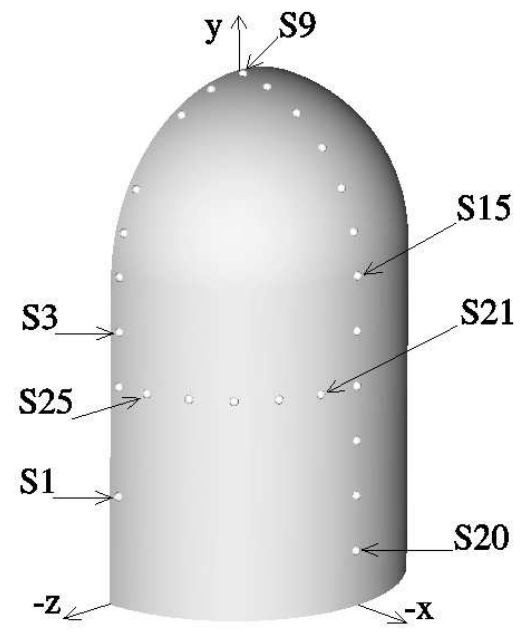

(a) Monitor distribution over the front side

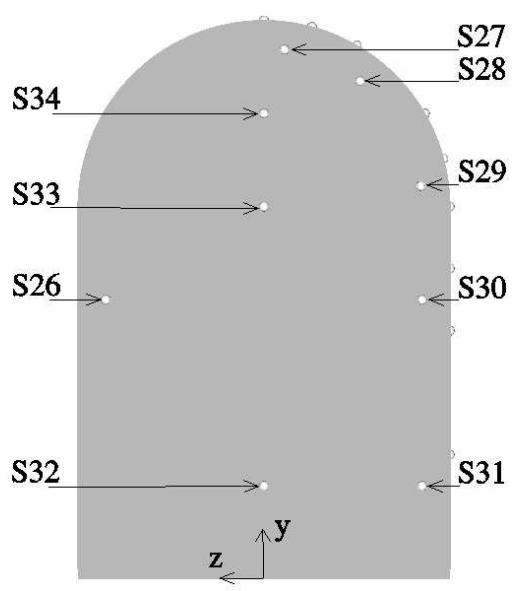

(b) Monitor distribution over the rear side

Figure 7. Location of surface sensors over mirror front and rear side respectively

scales in the wake reaches the rear side of the mirror and causes this appearance. The lack of high frequency content in the DES force coefficient can also be understood from Fig. 10(a) compared to Fig. 11(a) where the rms levels of the pressure are presented and supports the findings above.

Figures 10(b) and 11(b), gives the rms of the pressure fluctuations over the plate for the two cases. The figures shows that the shear layers over the cylindrical part of the mirror are the dominating cause to the sound generation. Comparing Figs. 11(a) and 11(b) reveals that the rms levels of the pressure fluctuations over the mirror rear side compared to the maximum values over the plate are ten times as small. For the LES case the corresponding maximum levels are approximately twice as large over the plate as over the mirror rear side, Figs. 10(a) and 10(b). This discrepancy will have a significant effect on the sound radiation. Still, even though the levels for the DES case are low over the mirror rear side both cases show similar results over 


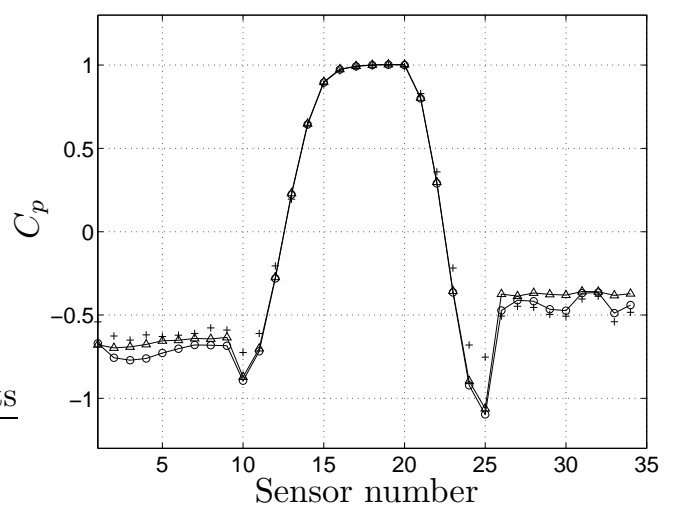

Figure 8. Mean pressure over mirror, (+) Measured and (॰) LES ( $\triangle$ ) DES

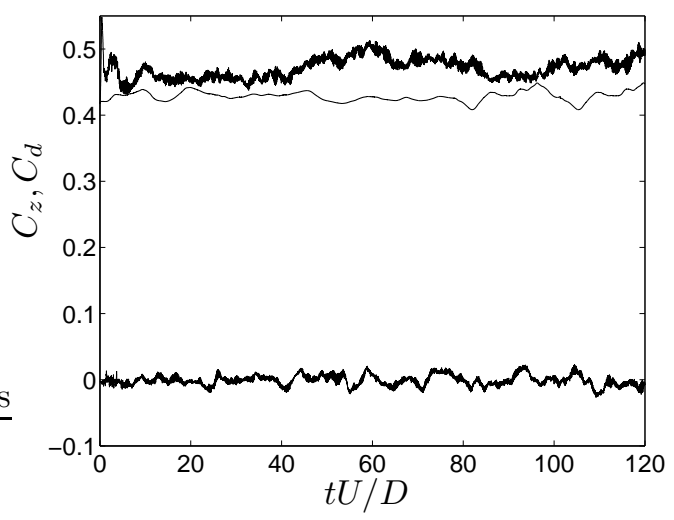

Figure 9. Force coefficients, $C_{d}$ LES (highest amplitude) and DES, $C_{z}$ LES

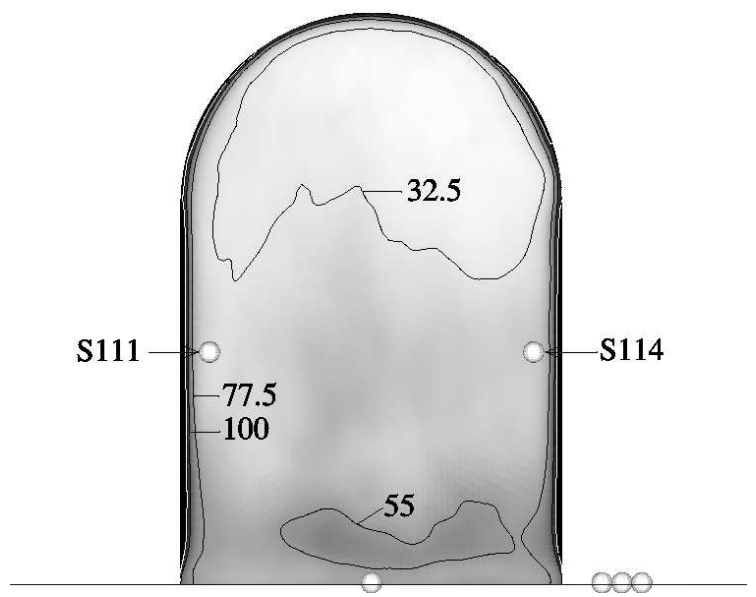

(a) RMS of pressure on mirror rear side, LES

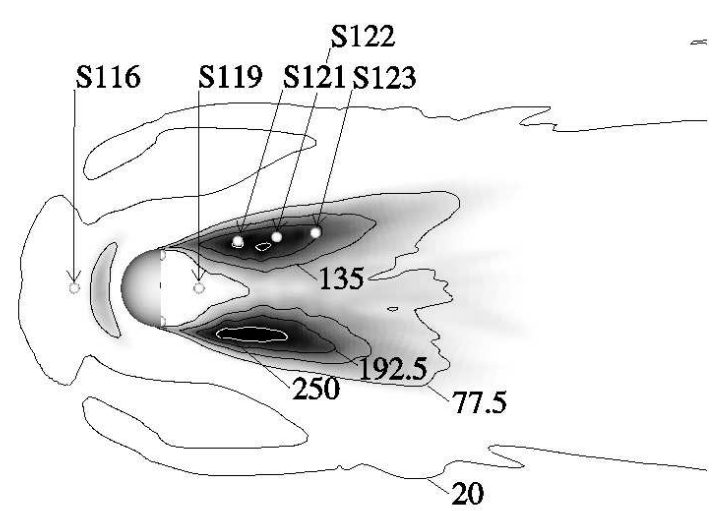

(b) RMS of pressure fluctuations over the plate, LES

Figure 10. RMS of wall pressure and sensor positions, LES

the plate. Next, pressure fluctuations returned by instantaneous piezo sensors as well as the corresponding computed signals are presented in the frequency plane. The locations of these sensors are given in Table A 


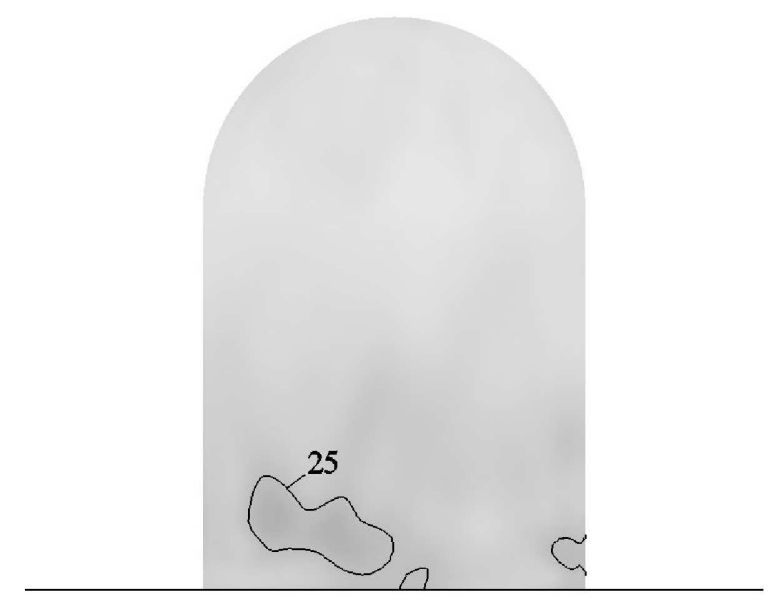

(a) RMS of pressure on mirror rear side, DES

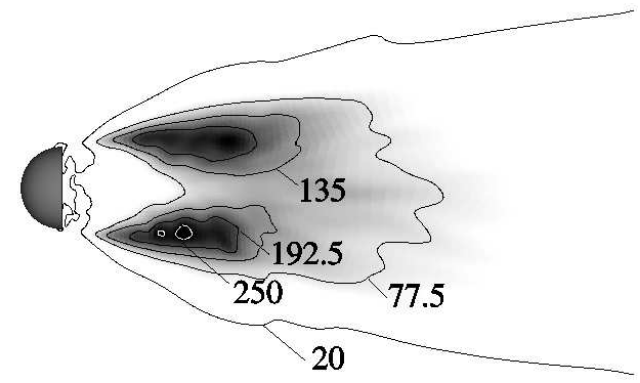

(b) RMS of pressure fluctuations over the plate, DES

Figure 11. RMS of wall pressure, DES

in Appendix A and in Fig. 10.

Analyzing discontinuous signals in the frequency domain is always a controversial matter, especially for short time sequences. For acoustic measurements the standard procedure is approximately seven seconds of sampled data to obtain converged statistics, which were not feasible with present state computer resources. The measured results obtained from Daimler-Chrysler [24] indicated that the signals were low pass filtered using a Butterworth filter with a stop-band frequency of $2000[\mathrm{~Hz}]$ and a bin width of $10[\mathrm{~Hz}]$. Here, the signals are also Butterworth filtered with the same stop-band frequency and spectral results are presented with the same bin width, $10[\mathrm{~Hz}]$. A second order filter is used in the present work and the bin width is reconstructed through integrating the spectra over $10[\mathrm{~Hz}]$ blocks. The wall pressures extracted from the simulations are sampled each time step for both cases corresponding to a sampling frequency of $50[\mathrm{kHz}]$.

In Figs. 12(a) to 14(b) the resolved cut-off frequency is marked by a vertical line. This cut-off frequency is estimated as $f_{\max }=\left|u_{p, r m s}\right| /\left(2 \Delta x_{p}\right)$, where $u_{p, r m s}$ is the rms of the resolved fluctuating velocity magnitude parallel to the wall and $\Delta x_{p}$ is the local grid spacing projected in the $u_{p, r m s}$-direction. The resolved cut-off frequencies for the first off-wall cells over the wall pressure sensors are summarized in Table 1.

\begin{tabular}{|c|c|c|c|c|c|}
\hline \hline \multicolumn{5}{|c|}{ Cut-off frequency for first off-wall cells } \\
\hline Sensor & $\left|u_{p, r m s}\right|$, LES & $\left|u_{p, r m s}\right|$, DES & $2 \Delta x_{p}$ & $f_{\text {max }}$, LES & $f_{\text {max }}$, DES \\
\hline S111 & 6.18 & 3.23 & 0.0121 & 511 & 267 \\
\hline S114 & 6.22 & 3.15 & 0.0121 & 514 & 260 \\
\hline S116 & 5.76 & 0.05 & 0.0263 & 219 & 1.90 \\
\hline S119 & 9.41 & 8.17 & 0.0192 & 490 & 425 \\
\hline S121 & 16.1 & 11.8 & 0.0240 & 671 & 492 \\
\hline S122 & 17.3 & 13.0 & 0.0244 & 709 & 533 \\
\hline S123 & 14.2 & 14.8 & 0.0244 & 582 & 606 \\
\hline \hline
\end{tabular}

Table 1. Cut-off frequency for the surface sensors

For an automotive applications the wall pressure levels is by far the most important result. The wall pressure represents the physical excitation of the exterior structure and from the driver or passengers point of view the radiated sound at a location outside the compartment is of less interest. The fluctuating pressure 
level presented in the figures is computed in the following way

$$
P L=10 \log _{10} \frac{\hat{p}}{p_{\text {ref }}^{2}}
$$

where $p_{\text {ref }}=2 \cdot 10^{-5}[\mathrm{~Pa}]$ is the reference pressure and $\hat{p}$ is power spectral density of the fluctuating pressure. The presentation of hydrodynamic pressure fluctuations in terms of SPL can be somewhat misleading as sound pressure levels only account for emissions in the farfield. For this reason, the power density spectrum of fluctuating hydrodynamic pressure levels is denoted as Pressure Levels (PL) instead. Figures 12 to 14 present the computed results as well as the Daimler-Chrysler measurements [24] for comparison.

The results collected from the two sensors located on the rear side of the mirror are presented in Figs. 12(a) to 12(d). From these figures it is clear that the DES model underpredict the source levels over the whole frequency range while the LES models predicts the levels fairly accurate all the way up to the resolved cut-off frequency. Above the resolved cut-off frequency several peaks in the LES signals are seen which may be due to aliasing effects. For the wall pressure levels this is of no concern since it occurs outside the valid frequency range. For sensor $S 116$ presented in Figs. 12(e) and 12(f) the discrepancy between the two simulations is even more pronounced. The location of this sensor is close to the horse-shoe vortex upstream the mirror and a misrepresentation in size and location can cause the discrepancy in results. The misrepresentation at this location is however expected due to the low resolution over the plate. The DES case show for this location significantly less fluctuations and the whole spectra in Fig. 12(f) is above the resolved cut-off frequency and contains thus only numerical noise. A possible cause is the RANS treatment of the boundary layers preventing fluctuations to occur. The trends in results for sensors $S 111-S 114$ can also be found at sensor $S 119$ located in the $z=0$ plane behind the mirror but to a much lesser extent. For the sensors located in the high level region behind the mirror $S 121-S 123$ the DES case show similar results as the LES case in the low frequency region although drops rapidly when reaching the resolved cut-off frequency. If this is caused by the discretization scheme or due to the turbulence model or a combination of both is unclear and should be investigated further. The grid size in this region is indeed coarse, approximately 0.06D and makes the results very promising for future work.

A general trend for the wall pressures results is that the levels are underpredicted by the DES model but overpredicted with the LES approach. An overprediction would intuitively be expected due to the incompressible assumption.

\section{Acoustic results}

The sound emission at five observer points is computed and compared with experimental data. The positions of the observers are given in Table A in Appendix A and are shown in Fig. 15. Neither the locations of the microphones nor the measured results were given in advance, the measured results and their positions were therefore taken from the work of Rung et al. [3]. In this section the same Butterworth filter and bin width are used as in the previous section but the stop-band frequency was reduced to $400[\mathrm{~Hz}]$ and added to the figures as solid vertical lines.

The storage of the two surface integral terms in Eq. 1, contributes to 8.3GB of data for the DES simulation. Therefore the sampling frequency for the LES case were changed to every second sample. Due to the wide frequency range between the stop-band of the filter and the sample frequency it was assumed that this shift in sampling rate not would change the outcome of the results significantly.

For observer $M 1$, located upstream of and above the mirror, the DES results matches the measurements, Fig. 16(b). The LES case on the other hand shows a clear overprediction over the whole frequency range, Fig. 16(a). This is in contradiction to the findings in the wall pressure section. An explanation to this appearance may be due to contamination by unresolved fluctuations over the plate which are used to compute the radiated sound. The resolved cut-off frequency computed in the previous section differs widely in frequency range. When conducting the surface integration over the plate, pressure fluctuations well resolved or not will be included in this integration and will give an erroneous result. The DES case on the other hand dampens turbulent fluctuation due to an excessive production of turbulent viscosity. This was found both for the force coefficient as well as wall pressure signals. Over the plate the flow corresponds to an almost RANS-like kind of flow with a low level of fluctuations. This is one explanation to why the SPL at the observers is much lower compared to the LES case. For observers $M 4, M 10, M 11$ the results show similar trends, Figs. 16(c) and 17 (b). Slightly different is however the result obtained for observer $M 14$. The low frequency levels in 


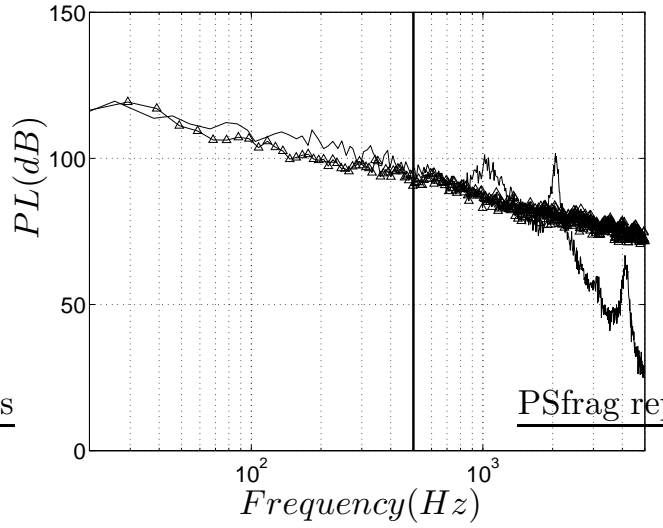

(a) PL at surface sensor $\mathrm{S} 111,(\triangle)$ Measured, (-) LES

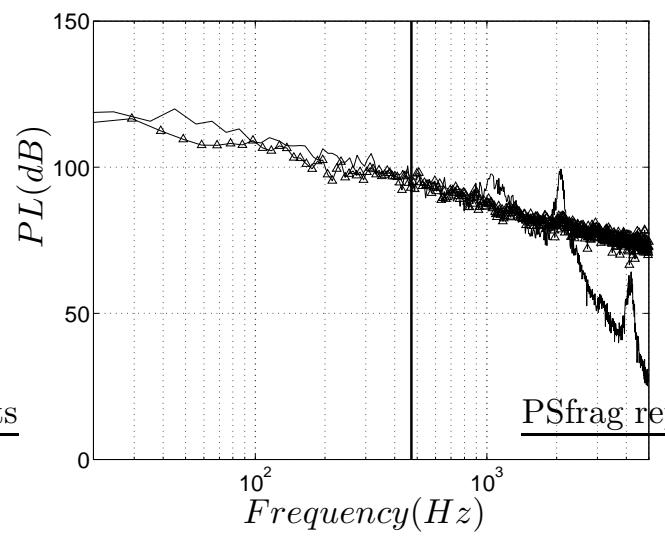

(c) PL at surface sensor S114, $(\triangle)$ Measured, (-) LES

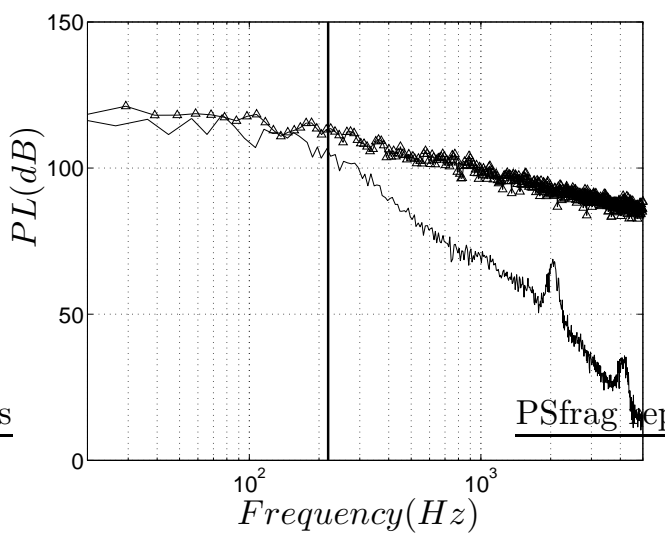

(e) PL at surface sensor S116, $(\triangle)$ Measured, (-) LES

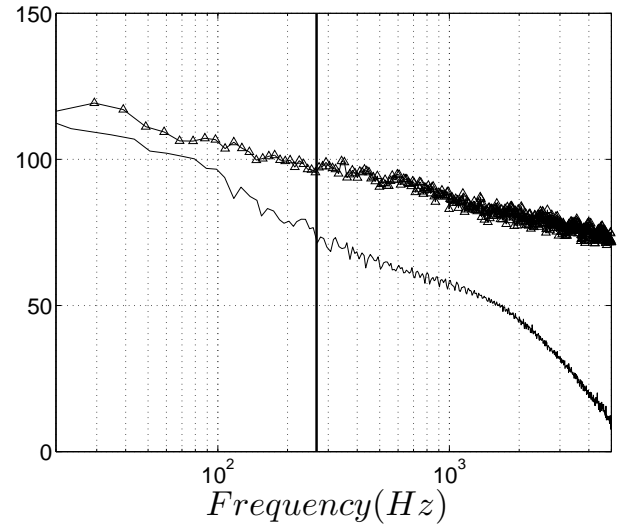

(b) PL at surface sensor S111, $(\triangle)$ Measured, (-) DES

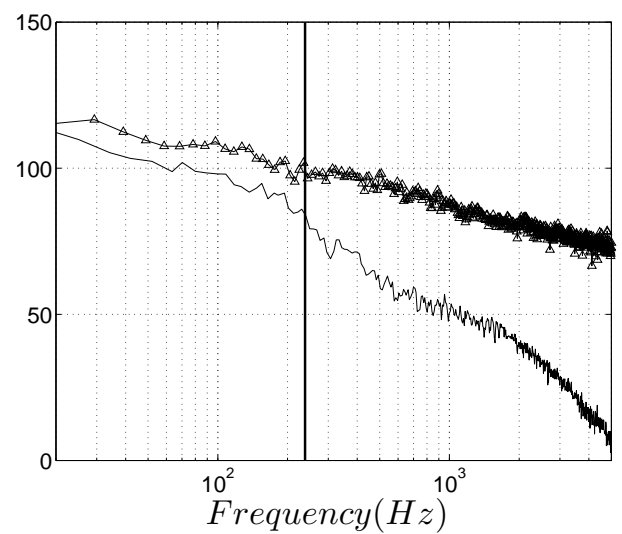

(d) PL at surface sensor S114, $(\triangle)$ Measured, (-) DES

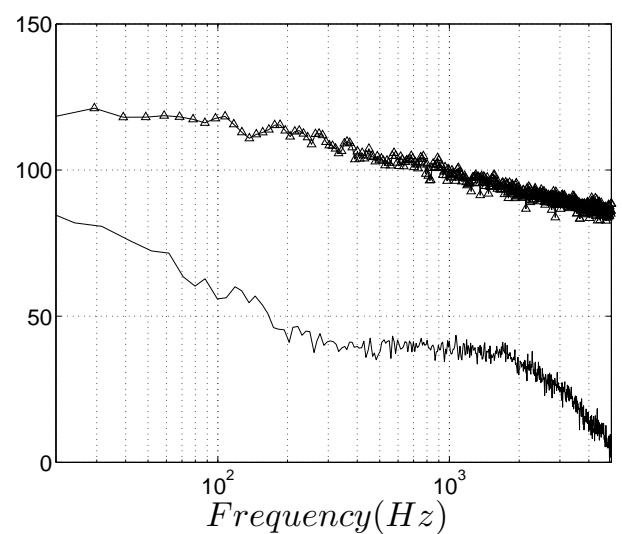

(f) PL at surface sensor S116, $(\triangle)$ Measured, (-) DES

Figure 12. PL at sensors S111 to S116 


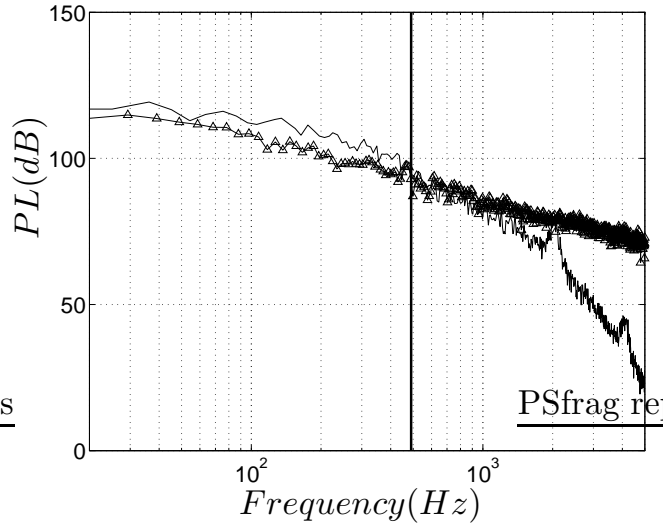

(a) PL at surface sensor $\mathrm{S} 119,(\triangle)$ Measured, (-) LES

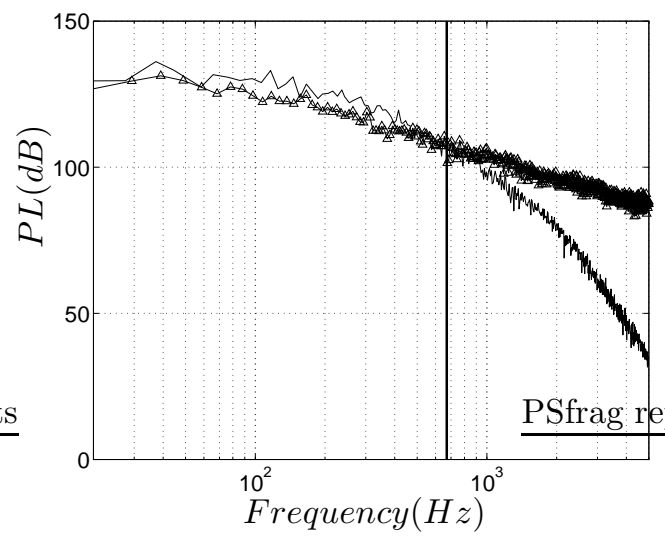

(c) PL at surface sensor S121, $(\triangle)$ Measured, (-) LES

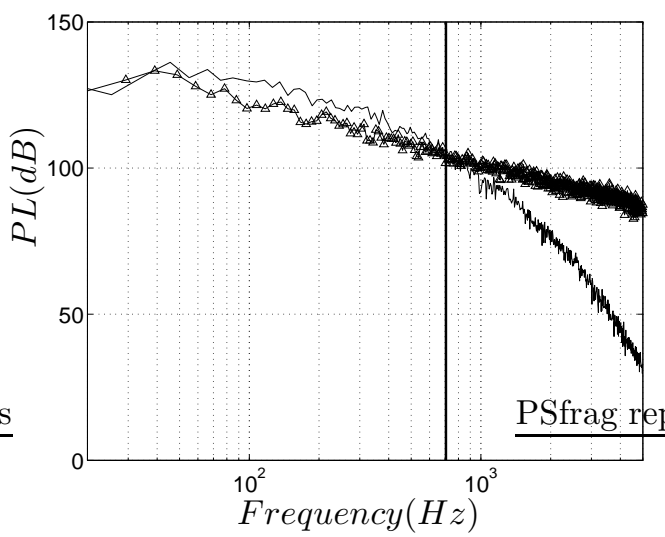

(e) PL at surface sensor S122, $(\triangle)$ Measured, (-) LES

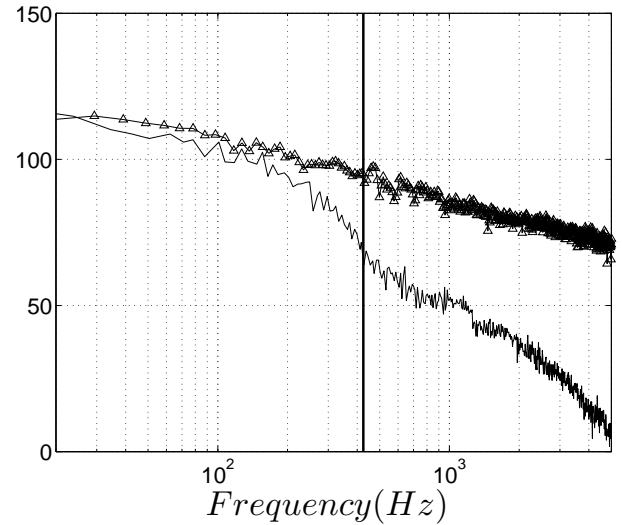

(b) PL at surface sensor S119, $(\triangle)$ Measured, (-) DES

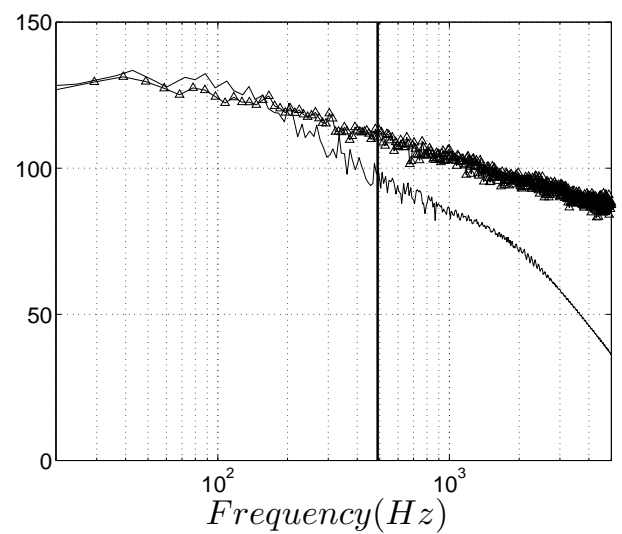

(d) PL at surface sensor S121, $(\triangle)$ Measured, (-) DES

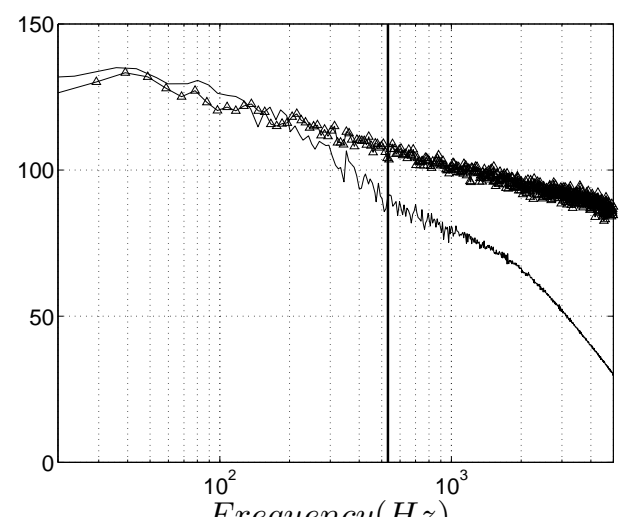

(f) PL at surface sensor S122, $(\triangle)$ Measured, (-) DES

Figure 13. PL at sensors S119 to S122 


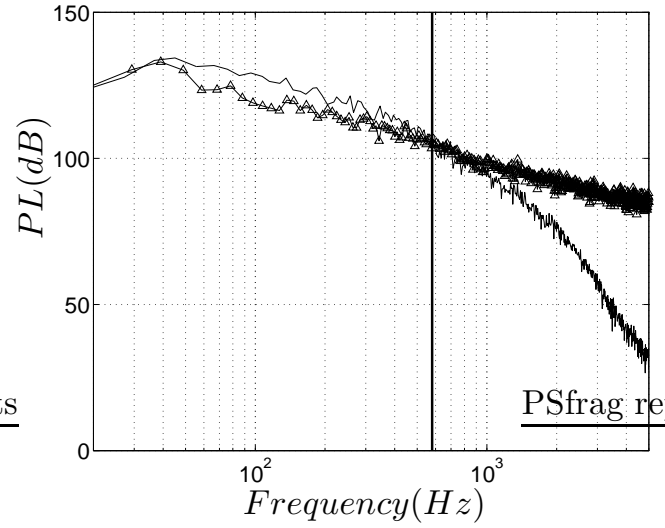

(a) PL at surface sensor $\mathrm{S} 123,(\triangle)$ Measured, (-) LES

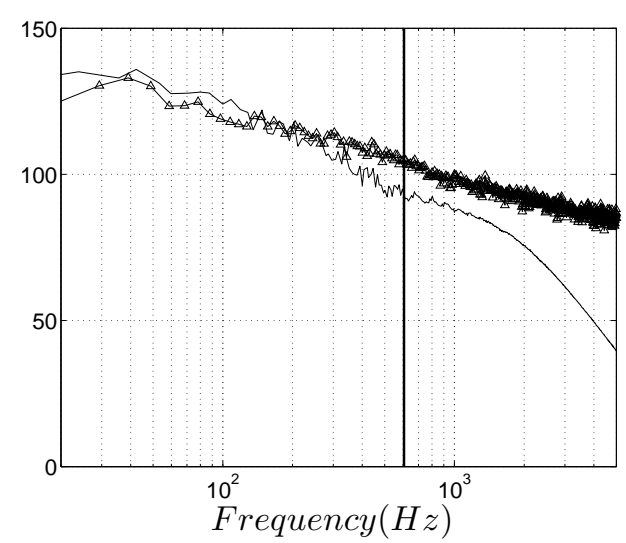

(b) PL at surface sensor S123, $(\triangle)$ Measured, (-) DES

Figure 14. PL at sensors S123

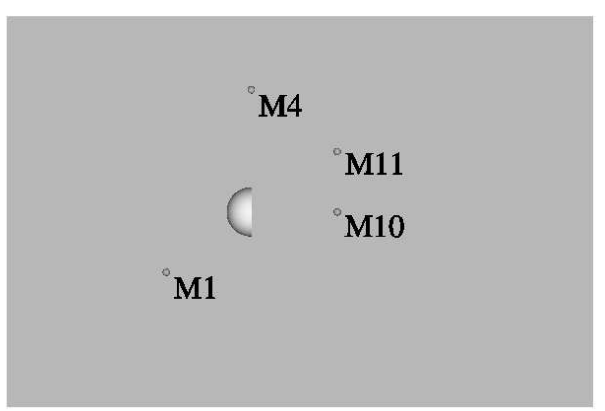

(a) Microphone position top view

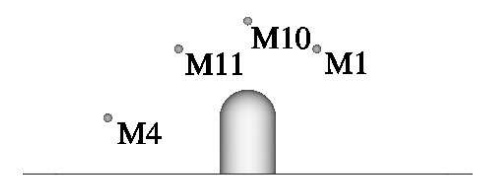

(b) Microphone position front view

Figure 15. Microphone positions, top and front view respectively

SPL for both simulations are clearly underpredicted for this observer, Figs. 17(c) and 17(d). The cause can be of experimental character due to a possible masking of the signal by influences of the wind tunnel. To investigate this further, the background noise of the tunnel must be known at the present freestream velocity.

\section{Conclusions and future work}

In this paper a first attempt is made to predict both the flow field and emitted sound past a generic side mirror mounted on a flat plate. The Reynolds number is $5.2 \cdot 10^{5}$ based on mirror diameter; the corresponding Mach number for this flow field is $M a=0.11$. Owing to the low Mach number, the flow field is solved on the basis of an assumption of incompressibility.

The present simulation attempts to capture this flow field by two different approaches using the same mesh. The first is by using the DES model and in the second approach the dynamic Smagorinsky model is used. The radiated sound is calculated from the Ffowkes-Williams and Hawkings analogy, where the two terms treated are the fluctuating pressure and its temporal derivative. Thus, two major assumptions are 


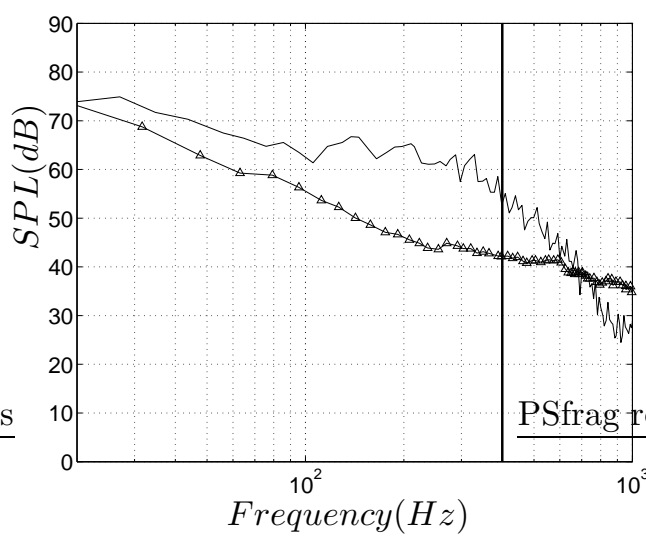

(a) SPL at microphone no. $1,(\triangle)$ Measured, (-) LES

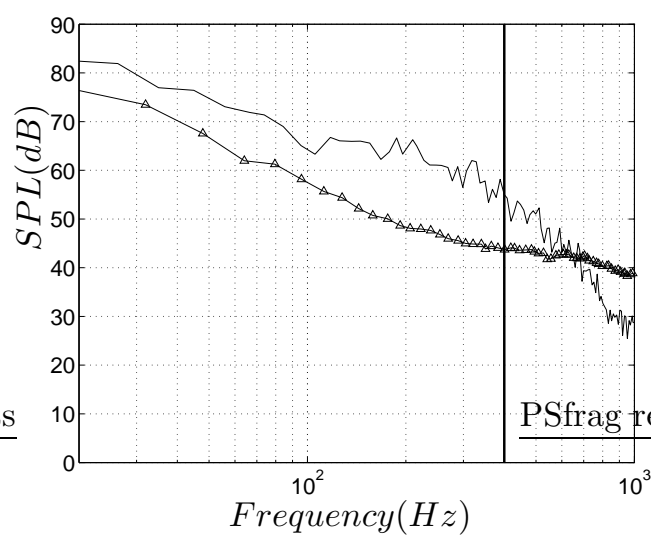

(c) SPL at microphone no. $4,(\triangle)$ Measured, (-) LES

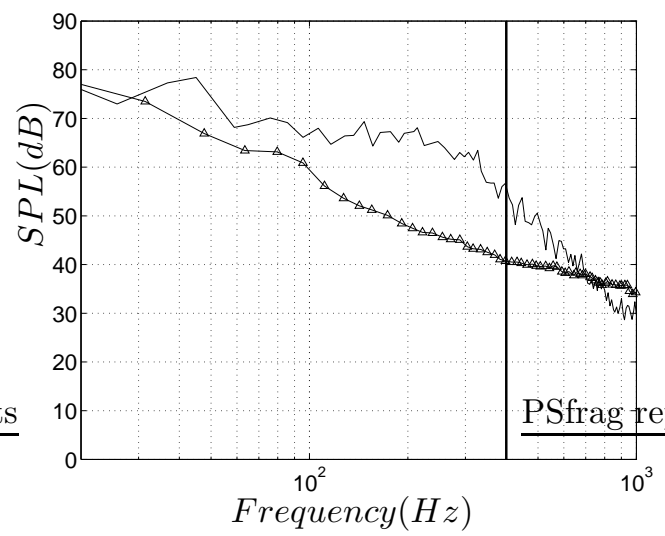

(e) SPL at microphone no. 10, $(\triangle)$ Measured, (-) LES

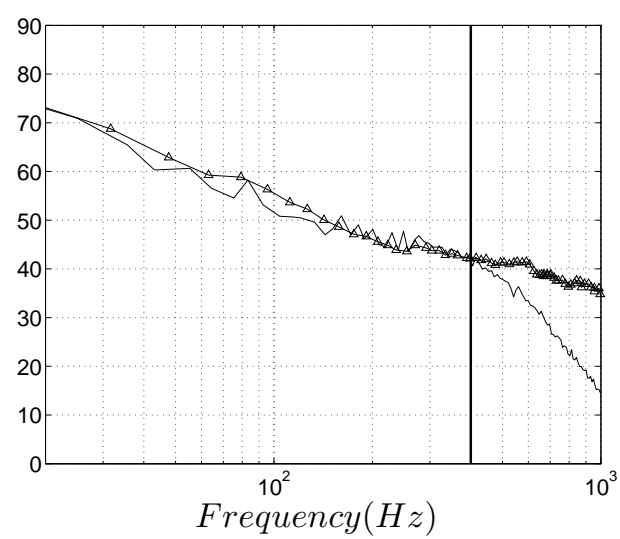

(b) SPL at microphone no. $1,(\triangle)$ Measured, $(-)$ DES

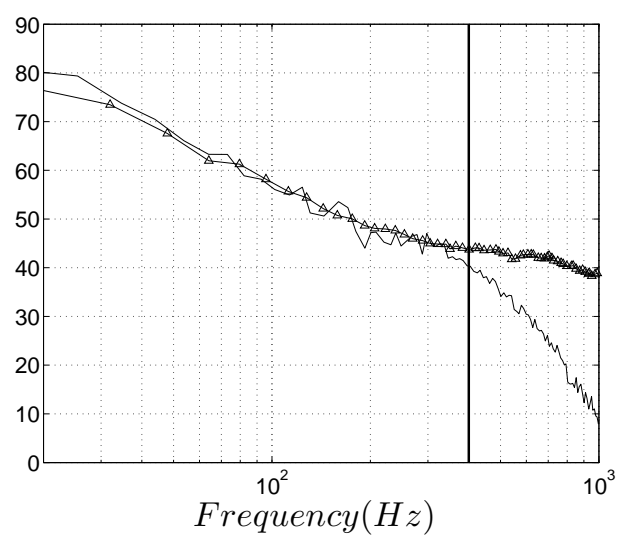

(d) SPL at microphone no. 4, $(\triangle)$ Measured, (-) DES

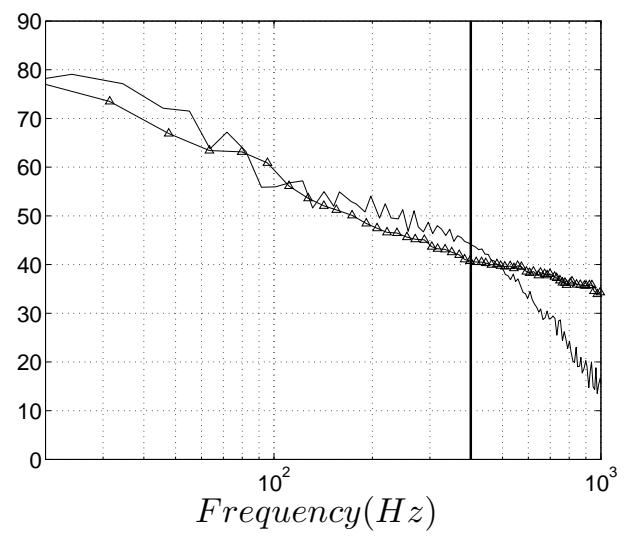

(f) SPL at microphone no. 10, $(\triangle)$ Measured, (-) DES

Figure 16. SPL at observers M1 to M10 


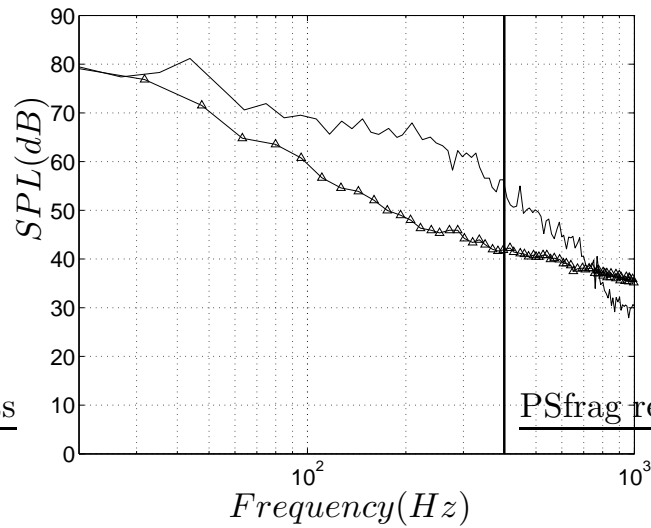

(a) SPL at microphone no. 11, $(\triangle)$ Measured, (-) LES

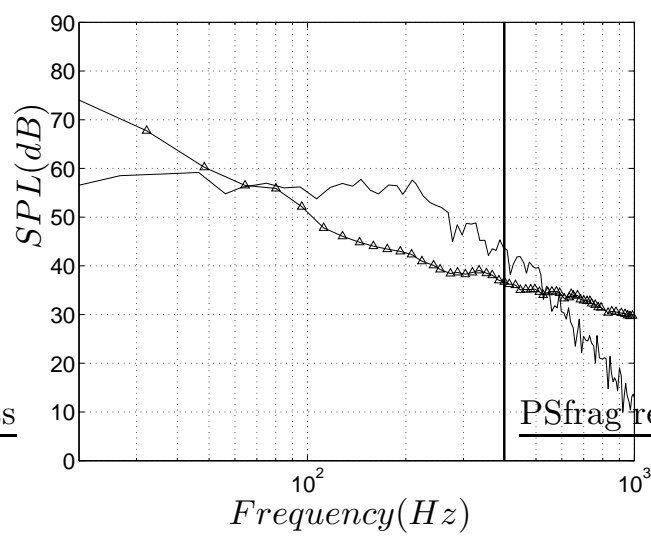

(c) SPL at microphone no. 14, $(\triangle)$ Measured, (-) LES

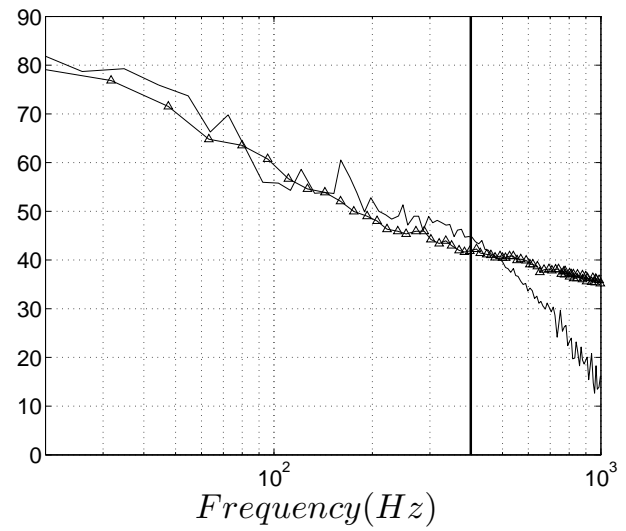

(b) SPL at microphone no. 11, $(\triangle)$ Measured, (-) DES

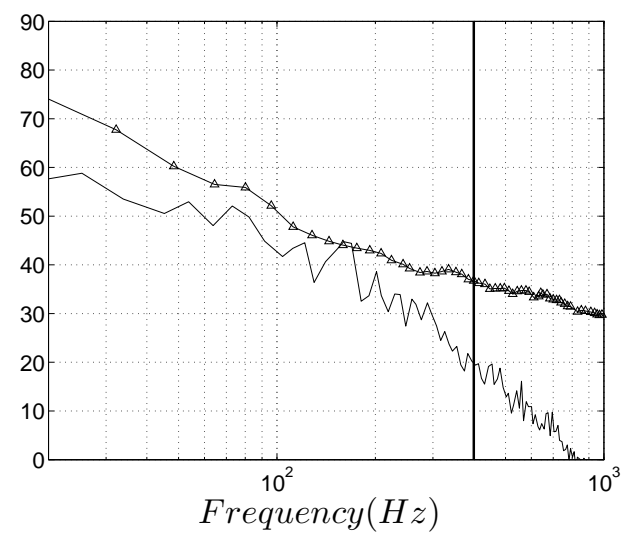

(d) SPL at microphone no. 14, $(\triangle)$ Measured, (-) DES

Figure 17. SPL at observers M11 to M14 
made. First, quadrapole terms are neglected and secondly the simulation is conducted in an incompressible manner, which neglects any sound wave reflection. The overall conclusions drawn from the present simulation are as follows.

- A conservative approach to estimate resolved cut-off frequency is presented and used for primarily the wall pressure levels.

- The LES simulation predicts the wall pressure fluctuations accurately up to the resolved cut-off frequency and shows that even a coarse LES is a better candidate for this kind of flow compared to the DES model.

- A drawback with the LES approach at present state is that also unresolved fluctuations are treated in the acoustic surface integral. This could possibly be improved by filtering the wall pressure fluctuations locally before conducting the surface integration.

- Major discrepancies in the radiated sound are found for the most distant observer, possibly due to the background noise of the tunnel.

\section{Acknowledgments}

This work was supported by the Volvo Car Corporation, the Swedish Agency for Innovation Systems (VINNOVA) and FLUENT Sweden. We also would express our gratitude toward Dr. Franz R. Klimetzek at Daimler Chrysler, who let us use their experimental data and pictures.

\section{References}

${ }^{1}$ Höld, R., Brenneis, A., and Eberle, A., "Numerical Simulation of Aeroacoustic Sound Generated by Generic Bodies Placed on a Plate: Part I - Prediction of Aeroacoustic Sources," 5th AIAA/CEAS Aeroacoustics Conference. Seattle, Washington, 10-12 May 1999, pp. AIAA-99-1896.

${ }^{2}$ Siegert, R., Schwartz, V., and Reichenberger, J., "Numerical Simulation of Aeroacoustic Sound Generated by Generic Bodies Placed on a Plate: Part II - Prediction of Radiated Sound Pressure," 5th AIAA/CEAS Aeroacoustics Conference. Seattle, Washington, 10-12 May 1999, pp. AIAA-99-1895.

${ }^{3}$ Rung, T., Eschricht, D., Yan, J., and Thiele, F., "Sound Radiation of the Vortex Flow past a Generic Side Mirror," 8th AIAA/CEAS Aeroacoustics Conference. Breckenridge, Colorado, 17-19 June, 2002, pp. AIAA-2002-2340.

${ }^{4}$ Consortium, E., "Validation Update: Model Mirror," Aeroacoustic Consortium 2003/2004 Presentation, 20 August, 2003.

${ }^{5}$ Bipin, L., Sandeep, S., and Jieyong, X., "Computational Aeroacoustic Analysis of a Generic Side View Mirror," Noise and Vibration Conference and Exhibition. Traverse City, Michigan, 5-8 May, 2003, pp. SAE-2003-01-1698.

${ }^{6}$ Ffowcs Williams, J. and Hawkings, D., "Sound generation by turbulence and surfaces in arbitrary motion," Philos. Trans. Roy. Soc., Vol. A 264 No. 1151, 1969, pp. 321-342.

${ }^{7}$ Brentner, K. and Farassat, F., "Modeling Aerodynamically Generated Sound of Helicopter Rotors," Prog. Aerospace Sci., Vol. 39, 2003, pp. 83-120.

${ }^{8}$ Spalart, P., Jou, W.-H., Strelets, M., and Allmaras, S., "Comments on the feasibility of LES for wings, and on a hybrid RANS/LES approach," 1st AFOSR International conference on DNS/LES. In advances in DNS/LES, 1997, pp. 137-147.

${ }^{9}$ Travin, A., Shur, M., Strelets, M., and Spalart, P., "Detached-Eddy Simulations Past a Circular Cylinder," Flow, Turbulence and Combustion, Vol. 63, 1999, pp. 293-313.

${ }^{10}$ Strelets, M., "Detached Eddy Simulation of Massively Separated Flows," 39th AIAA Aerospace Sciences Meeting and Exhibit. Reno, NV, 8-11 January, 2001, pp. AIAA-2001-0879.

${ }^{11}$ Vatsa, V. and Singer, B., "Evaluation of a Second-Order Accurate Navier-Stokes Code for Detached Eddy Simulation Past a Circular Cylinder," 21th AIAA Aerospace Sciences Meeting and Exhibit. Reno, NV, 8-11 January, 2001, pp. AIAA2001-0879.

${ }^{12}$ Schmidt, S. and Thiele, F., "Detached Eddy Simulation of Flow around A-Airfoil," Flow, Turbulence and Combustion, Vol. 71, 2003, pp. 261-278.

${ }^{13}$ Constantinescu, G. and Squires, K., "LES and DES Investigations of Turbulent Flow over a Sphere at Re=10000," Flow, Turbulence and Combustion, Vol. 70, 2003, pp. 267-298.

${ }^{14}$ Constantinescu, G. and Squires, K., "Numerical Investigations of Flow over a Sphere in the Subcritical and Supercritical Regimes," Physics of Fluids, Vol. 16, 2004, pp. 1449-1466.

${ }^{15}$ Shur, M., Spalart, P., Strelets, M., and Travin, A., "Navier-Stokes Simulation of Shedding Turbulent Flow Past a Circular Cylinder and a Cylinder with Backward Splitter Plate," Computational Fluid Dynamics '96, Proceedings of the Third ECCOMAS Computational Fluid Dynamics Conference, 9-13 September, 1996, pp. 676-682.

${ }^{16}$ Spalart, P., "Strategies for turbulence modelling and simulations," International Journal of Heat and Fluid Flow, Vol. 21, 2000, pp. 252-263.

${ }^{17}$ Germano, M., Piomelli, U., Moin, P., and Cabot, H., "A dynamic subgrid-scale eddy viscosity model," Physics of Fluids, Vol. A 3 (7), 1991, pp. 1760-1765. 
${ }^{18}$ Lilly, D., "A proposed modification of the Germano subgrid-scale closure method," Physics of Fluids, Vol. A 4 (3), 1992, pp. 633-635.

${ }^{19}$ Curle, N., "The influence of solid boundaries upon aerodynamic sound," Proc. Roy. Soc, Vol. A231, 1955, pp. 505-514.

${ }^{20}$ Ask, J. and Davidson, L., "An acoustic analogy applied to the laminar upstream flow over an open 2D cavity," In press, C.R. Mecanique, 2005.

${ }^{21}$ Ask, J. and Davison, L., "An investigation of outlet boundary conditions for incompressible near field acoustics," 11th AIAA/CEAS Aeroacoustics Conference, 23-25 May, 2005, pp. AIAA-2005-2992.

${ }^{22}$ Larsson, J., "Computational Aero Acoustics for Vehicle Applications," Chalmers University of Technology, 2002.

${ }^{23}$ Schlichting, H., Boundary Layer Theory, Springer, 1969.

${ }^{24}$ Klimetzek, F., "Personal communication," Daimler-Chrysler, Research and Technology, Advanced Concepts (RBP/BC), Sept, 2005.

${ }^{25}$ Spalart, P., Hedges, L., Shur, M., and Travin, A., "Simulation of Active Flow Control on a Stalled Airfoil," Flow, Turbulence and Combustion, Vol. 71, 2003, pp. 361-373.

${ }^{26}$ Hedges, L., Travin, A., and Spalart, P., "Detached-Eddy Simulations Over a Simplified Landing Gear," Journal of Fluids Engineering, Vol. 124, 2002, pp. 413-423.

${ }^{27}$ Menter, F., Kuntz, M., and Bender, R., "A scale-adaptive simulation model for turbulent flow predictions," 41st Aerospace Science Meeting and Exhibit, 6-9 January, 2003, pp. AIAA-2003-0767. 


\section{A. Microphone and Sensor positions}

\begin{tabular}{|c|c|c|c|}
\hline \hline \multicolumn{4}{|c|}{ Positions of microphones } \\
\hline Mic Id & $\mathrm{x}$ & $\mathrm{y}$ & $\mathrm{z}$ \\
\hline $\mathrm{M} 1$ & $-1.24 \mathrm{D}$ & $2.23 \mathrm{D}$ & $1.2345 \mathrm{D}$ \\
\hline $\mathrm{M} 4$ & $0.5 \mathrm{D}$ & $1 \mathrm{D}$ & $-2.5 \mathrm{D}$ \\
\hline $\mathrm{M} 10$ & $2.265 \mathrm{D}$ & $2.729 \mathrm{D}$ & 0 \\
\hline $\mathrm{M} 11$ & $2.265 \mathrm{D}$ & $2.23 \mathrm{D}$ & $-1.2345 \mathrm{D}$ \\
\hline $\mathrm{M} 14$ & $0.5 \mathrm{D}$ & $8.075 \mathrm{D}$ & $-7.1725 \mathrm{D}$ \\
\hline \hline
\end{tabular}

\begin{tabular}{|c|c|c|c|}
\hline \hline \multicolumn{4}{|c|}{ Positions of instantaneous pressure sensors } \\
\hline Senor Id & $\mathrm{x}$ & $\mathrm{y}$ & $\mathrm{z}$ \\
\hline $\mathrm{S} 111$ & $0.5 \mathrm{D}$ & $0.61 \mathrm{D}$ & $0.425 \mathrm{D}$ \\
\hline $\mathrm{S} 114$ & $0.5 \mathrm{D}$ & $0.61 \mathrm{D}$ & $-0.425 \mathrm{D}$ \\
\hline $\mathrm{S} 116$ & $-0.6 \mathrm{D}$ & 0 & 0 \\
\hline $\mathrm{S} 119$ & $1 \mathrm{D}$ & 0 & 0 \\
\hline $\mathrm{S} 121$ & $1.4945 \mathrm{D}$ & 0 & $-0.6045 \mathrm{D}$ \\
\hline $\mathrm{S} 122$ & $1.992 \mathrm{D}$ & 0 & $-0.657 \mathrm{D}$ \\
\hline $\mathrm{S} 123$ & $2.489 \mathrm{D}$ & 0 & $-0.709 \mathrm{D}$ \\
\hline \hline
\end{tabular}




\begin{tabular}{|c|c|c|c|}
\hline \hline \multicolumn{4}{|c|}{ Positions of mean pressure sensors over mirror } \\
\hline Sensor Id & $\mathrm{x}$ & $\mathrm{y}$ & $\mathrm{z}$ \\
\hline S1 & $0.468 \mathrm{D}$ & $0.3335 \mathrm{D}$ & $-0.499 \mathrm{D}$ \\
\hline S2 & $0.468 \mathrm{D}$ & $0.6665 \mathrm{D}$ & $-0.499 \mathrm{D}$ \\
\hline S3 & $0.468 \mathrm{D}$ & $0.8335 \mathrm{D}$ & $-0.499 \mathrm{D}$ \\
\hline S4 & $0.468 \mathrm{D}$ & $1 \mathrm{D}$ & $-0.499 \mathrm{D}$ \\
\hline S5 & $0.468 \mathrm{D}$ & $1.129 \mathrm{D}$ & $-0.482 \mathrm{D}$ \\
\hline S6 & $0.468 \mathrm{D}$ & $1.2495 \mathrm{D}$ & $-0.432 \mathrm{D}$ \\
\hline S7 & $0.468 \mathrm{D}$ & $1.432 \mathrm{D}$ & $-0.2495 \mathrm{D}$ \\
\hline S8 & $0.468 \mathrm{D}$ & $1.482 \mathrm{D}$ & $-0.129 \mathrm{D}$ \\
\hline S9 & $0.468 \mathrm{D}$ & $1.499 \mathrm{D}$ & 0 \\
\hline S10 & $0.3705 \mathrm{D}$ & $1.483 \mathrm{D}$ & 0 \\
\hline S11 & $0.25 \mathrm{D} 0$ & $1.433 \mathrm{D}$ & 0 \\
\hline S12 & $0.1465 \mathrm{D}$ & $1.3535 \mathrm{D}$ & 0 \\
\hline S13 & $0.067 \mathrm{D}$ & $1.25 \mathrm{D}$ & 0 \\
\hline S14 & $0.017 \mathrm{D}$ & $1.1295 \mathrm{D}$ & 0 \\
\hline S15 & 0 & $1 \mathrm{D}$ & 0 \\
\hline S16 & 0 & $0.833 \mathrm{D}$ & 0 \\
\hline S17 & 0 & $0.6665 \mathrm{D}$ & 0 \\
\hline S18 & 0 & $0.5 \mathrm{D}$ & 0 \\
\hline S19 & 0 & $0.3335 \mathrm{D}$ & 0 \\
\hline S20 & 0 & $0.1665 \mathrm{D}$ & 0 \\
\hline S21 & $0.017 \mathrm{D}$ & $0.6665 \mathrm{D}$ & $-0.1295 \mathrm{D}$ \\
\hline S22 & $0.067 \mathrm{D}$ & $0.6665 \mathrm{D}$ & $-0.25 \mathrm{D}$ \\
\hline S23 & $0.1465 \mathrm{D}$ & $0.6665 \mathrm{D}$ & $-0.3535 \mathrm{D}$ \\
\hline S24 & $0.25 \mathrm{D}$ & $0.6665 \mathrm{D}$ & $-0.433 \mathrm{D}$ \\
\hline S25 & $0.3705 \mathrm{D}$ & $0.6665 \mathrm{D}$ & $-0.483 \mathrm{D}$ \\
\hline S26 & $0.5 \mathrm{D}$ & $0.75 \mathrm{D}$ & $0.425 \mathrm{D}$ \\
\hline S27 & $0.5 \mathrm{D}$ & $1.4215 \mathrm{D}$ & $-0.0555 \mathrm{D}$ \\
\hline S28 & $0.5 \mathrm{D}$ & $1.337 \mathrm{D}$ & $-0.2585 \mathrm{D}$ \\
\hline S29 & $0.5 \mathrm{D}$ & $1.0555 \mathrm{D}$ & $-0.4215 \mathrm{D}$ \\
\hline S30 & $0.5 \mathrm{D}$ & $0.75 \mathrm{D}$ & $-0.425 \mathrm{D}$ \\
\hline S31 & $0.5 \mathrm{D}$ & $0.25 \mathrm{D}$ & $-0.425 \mathrm{D}$ \\
\hline S32 & $0.5 \mathrm{D}$ & $0.25 \mathrm{D}$ & 0 \\
\hline S33 & $0.5 \mathrm{D}$ & $1 \mathrm{D}$ & 0 \\
\hline S34 & $0.5 \mathrm{D}$ & $1.25 \mathrm{D}$ & 0 \\
\hline \hline & & & \\
\hline
\end{tabular}

20 of 20 